Chimia 45 (1991) 221-227

(C) Schweiz. Chemiker-Verband; ISSN 0009-4293

\section{Chromatographic Methods and Transport of Chemicals in Soils}

\author{
Michal Borkovec $^{\mathrm{a}}$ ), Bernhard Buchter ${ }^{\mathrm{a}}$ ), Hans Stichera)*, Philippe Behra ${ }^{\mathrm{b}}$ ), and \\ Michel Sardinc)
}

Abstract. Transport of chemicals in soils and groundwater aquifers has become an increasingly important field in hydrology and soil science. Much progress in this field originates fromthe picture of the soil as a giant chromatography column. Therefore, one uses successfully concepts borrowed from chromatography theory. The applicability of such ideas for the description of the transport of chemicals in the field is reviewed.

\section{Introduction}

The growing concern about groundwater pollution confronts soil scientists and hydrologists with a new challenge: predicting the transport of chemicals in the subsurface zone and groundwater aquifers [1][2]. The pollutant sources vary widely. Well known examples include chlorinated organic solvents from industrial dump sites, heavy metals transported as fly ash from mining and smelting processes, hydrocarbons from leaky gas stations and oil tanks, and pesticides originating from intense agricultural production [3-5]. The chemicals accumulate on the soil surface and may be taken up by plants or be transported by erosion processes. Most often, however, the pollutants infiltrate into the subsurface zone, possibly undergo chemical reactions and move into the groundwater below. In particular, if the groundwater aquifer is already used as drinking water supply (or will be used in the future), its contamination must be taken very seriously.

Due to the heterogeneous nature of soils the transport and transformation processes of chemicals in the subsurface zone are quite complex. The prediction of the movement of a given contaminant is, therefore, a difficult task [1]. Some chemicals, typically inorganic anions such as chloride or nitrate, interact weakly with the soil matrix and move quite rapidly (maybe several meters per year) with the infiltrating water along. Depending on the type of soil, this movement may be extremely rapid and channeled by cracks or quite slow and far spreading [2]. Other

\footnotetext{
*Correspondence: Prof. H. Sticher

a) Institute of Terrestrial Ecology

Federal Institute of Technology

CH-8092 Zürich

b) Institute of Fluid Mechanics

Louis Pasteur University

URA CNRS 854

F-67100 Strasbourg

c) Chemical Engineering Science Laboratory

CNRS-ENSIC

F-54000 Nancy
}

chemicals, e.g. most heavy-metal cations, adsorb strongly on the soil matrix and often move extremely slowly (less than $1 \mathrm{~m}$ per century). However, such accumulated chemicals could be released, if another pollutant is involved, or if the climatic conditions change. A well known example is the concem that accumulated heavy metals might become mobilized by the input of protons from the acid rain [4].

Pollutants in soils often undergo various chemical reactions. Near the soil surface, which is in contact with air, oxidation reactions are common. On the other hand, in the anoxic groundwater region the chemicals mostly undergo a typical sequence of reduction reactions [5]. The processes are further complicated by precipitation or coprecipitation reactions which are often difficult to distinguish from adsorption processes. Other complications arise in the case of microbiologically controlled redox processes (biodegradation). In recent years, additional transport mechanisms became the focus of an active discussion. Examples involve diffusion of volatile chemicals in the gas phase of the soil matrix, two-phase flow of organic solvents not miscible with water, and transport of adsorbed chemicals by bacteria, organic matter or microscopic inorganic particles (i.e. colloids) [6-8].

Deciding on the reclamation procedure of a particular dump site or contaminated region, it is usually not possible to investigate the rate of transport and leaching of pollutants in situ. Transport of chemicals in soils happen on time scales of years or longer, and meaningful field experiments have to be planned carefully, require costly installations and sampling periods covering several years [9-11]. When such results become available, it may be already too late for an appropriate restoration procedure of the site. A more promising strategy consists in performing simple laboratory and short-term field experiments. The results of such experiments can be interpreted with an appropriate theoretical model which, in turn, can be used to obtain long-time predictions. Such predictions may significantly help in deciding upon a proper decontamination method of a particular site.

Unfortunately, this type of approach is still far from satisfactory. Although the understanding of the transport properties in soils has significantly improved during the last decade, in many cases it is still far from predictive. Especially, our predictive means are quite limited when dealing with chemicals interacting strongly with the soil matrix or undergoing chemical reactions. As a result of this situation, there is a pressing need to gather a better understanding of transport processes of chemicals in the subsurface zone and aquifers which would enable the prediction of the relevant features of chemical movement on a given site without the requirement of year long field experiments.

In this review, several new ideas in soilsolute transport are discussed [12-14]. Chemists may be intrigued to see how familiar concepts from chromatography are used to describe soil-solute transport. Similarities between these two fields are emphasized, but the specific aspects of field scale transport of chemicals and the limitations of such 'chromatographic' approach are also pointed out. First, we shall focus on the transport of non-interacting chemicals (conservative tracers) and then proceed with the discussion of the coupling between chemistry and transport. No attempt is made to review of this vast field completely, rather the underlying concepts are discussed on a comprehensive level, and a few illustrative examples are presented.

\section{Transport of Tracers}

Transport of chemically nonreactive and non-sorbing compounds (conservative tracers) in porous media has been studied during the last 40 years in the field of chromatography, hydrology, and chemical engineering. A classical example of such a process in a porous medium is a chromatography column. As a conservative tracer, one commonly uses a radioactively labeled water, chloride, bromide, or any other convenient non-sorbing ions. The discussion starts with the simplest case, where the chromatography column is randomly packed with nonporous, monodisperse, spherical beads. As long the flow velocity is very low, one expects that the convective (advective) transport and molecular diffusion (dispersion) simply superpose, and the concentration profile $c(x, t)$ of the tracer develops according to the linear convection-dispersion equation [15-19]

$$
\frac{\partial c}{\partial t}=D \frac{\partial^{2} c}{\partial x}-v \frac{\partial c}{\partial x}
$$

It turns out, that at higher flow velocities Eqn. 2.1 is still valid, but with a dispersion coefficient $D$ being much larger than the 
molecular diffusion coefficient with a typical value of $D_{m} \approx 10^{-5} \mathrm{~cm}^{2} / \mathrm{s}$. The dispersion coefficient increases roughly linearly with the velocity $v$ according to [1][16]

$$
D \approx D_{\mathrm{m}}+\lambda v
$$

The proportionality constant $\lambda$ has a dimension of length and is called the dispersivity. The true (interstitial) velocity $v$, on the other hand, is just $q$, the flux of the solven through the column (volume per unit time and area, Darcy velocity) divided by the porosity of the medium $\theta$, namely

$$
v=\frac{q}{\theta}
$$

To understand the reason why the convection-dispersion equation is still applicable at higher flow velocities, recall why Eqn 2.1 is valid at low velocities where molecular diffusion is the dominant dispersion mechanism [15]. Molecular diffusion originates from the random motion of molecules, which can be viewed as a succession of random jumps between neighboring solvent cages of approximately one molecule diameter $\xi$ with typical jump time (collision time) $\tau$. Such a random walk gives rise to a diffusion process with a molecular diffusion coefficient $D_{m} \approx \xi^{2} / \tau$. Increasing the flow velocity, one reaches a region where much more effective displacement takes over (mechanic dispersion). As the tracer molecules are carried by the flowing liquid, they now jump randomly between the 'holes' of the porous bed. The separation distance is comparable to the diameter of the spheres $d$ which gives the jump distance $\xi \approx d$ and the jump time $\tau \approx d / v$. One obtains a dispersion coefficient $D \approx \xi^{2} / \tau \approx d v$ and finds a dispersivity $\lambda \approx d$. This estimate holds quite accurately for randomly packed spheres of diameter $d$ in a laboratory column. It is essential to note, that the convection-dispersion equation (Eqn. 2.I) only applies to time and length scales much larger than the typical jump times and distances of the underlying random walk. In particular, it does not apply down the molecular scale for small velocities nor down to the scale of the particle diameter for higher velocities. This point will be important in the discussion of transport of tracers in the field where the characteristic times and distances are much larger.

Suppose that, at the time origin $(t=0)$ a small quantity of a conservative tracer is injected very quickly at the inlet of the chromatography column $(x=0)$ into the solvent flowing through the column. The concentration profile $c(x, t)$ of the tracer will be sharply peaked at the beginning and will slowly spread while moving through the column. Using Eqn. 2.l one can show that the center of mass of the peak will travel with the true velocity $v$ (see Eqn. 2.3)

$$
<x>=v t
$$

At the same time, the pulse will spread and its width can be characterized by the standard deviation which is related to the dispersion coefficient $D$ (see Eqn.2.2). The variance of the chromatography peak is given by

$$
<\delta x^{2}>=2 D t
$$

The peak travels faster than it broadens and the relative width of the peak, the coefficient of variation, decreases with time according to

$$
\frac{\left\langle\delta x^{2}\right\rangle}{\langle x\rangle^{2}}=\frac{2 D}{v^{2} t}
$$

In chromatography, one is usually not interested in the spatial distribution of the chemicals in the column at given time, but rather in the time dependence of the concentrations at the outlet of the column. Suppose a short concentration impulse is applied at $t$ $=0$ and the outflow concentration $c(L, t)$ is monitored. A typical response of the column is shown in Fig. Ia giving a concentration peak which arrives at the outlet after a mean time (characteristic time of the column)

$$
<t>=\frac{L}{v}
$$

Its relative spread in time is

$$
\frac{\left.<\delta t^{2}\right\rangle}{\langle t\rangle^{2}}=\frac{2 D}{v L}=\frac{2}{P e}
$$

Eqn. 2.8 introduces the dimensionless Peclet column number $P e=L v / D(P e / 2$ corresponds to the 'number of plates' in chromatography) [20]. The above relation reflects a well known fact: the longer the column, the sharper the chromatography peaks. Eqn. 2.8 can be obtained in a nonrigorous fashion from the approximate equality of the relative spread in time and space (see Eqn. 2.6). The rigorous discussion presented, e.g., in two recent textbooks [1][2], requires proper definitions of the boundary conditions and the concepts of residence and flux concentrations.

Because of the linearity of Eqn. 2.1, the concentration at the outflow of the column $c(L, t)$ is a linear functional of the input concentration $c(0, t)$, namely

$$
c(L, t)=\int_{0}^{t} g\left(t-t^{\prime}, L\right) c\left(0, t^{\prime}\right) d t^{\prime}
$$

The response function $g(t, L)$ corresponds physically to the residence-time distribution in the column [2][17]. In the case of pulse input, the concentration at the outlet of the column is nothing but the response function $g(t, L)$ itself. Thus, as long the column behaves linearly, knowing the response from one particular input profile, one may deduce the response of any given input profile by applying Eqn. 2.9. For the common input concentration profile of a sudden change of
CHIMIA 45 (1991) Nr. $7 / 8$ (Juli/August)

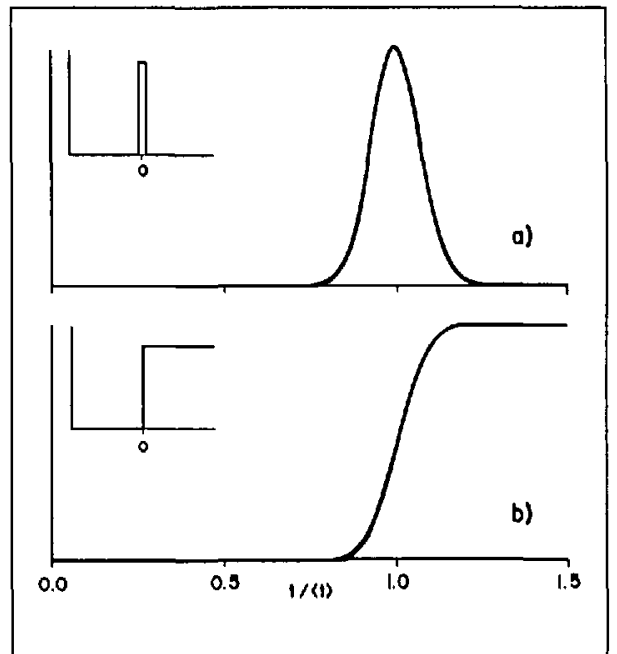

Fig. 1. Comparison of a chromatography column response with a Peclet number $\mathrm{Pe}=40$ for different input profiles (see inset). a) Pulse input, $b$ ) Step input.

concentration at $t=0$, one observes from Eqn. 2.9 that the concentration at the outlet is simply the integral of the response function. The situation is summarized in Fig. Ib. The abscissa in Fig. I, namely the variable $t /$ $\langle t>$ can also be interpreted as the cumulative volume of the effluent $V(t) / V_{0}$ normalized to the pore volume of the column $V_{0}$. The fact that $t /\langle t\rangle=V(t) / V_{0}$ follows from Eqn. 2.3 together with the relations $V(t)=q A t$ and $V_{0}$ $=\theta V_{c}\left(V_{c}\right.$ being the total volume of the column, $A$ its cross-sectional area, $V_{c}=L A$ ).

Particles used to fill chromatography columns are usually not compact but rather porous in order to obtain a larger surface area for the adsorption of the chemicals. The notions necessary for understanding transport in such a medium are very important in soils and also in the transport of chemicals. Hence, this process, which has been discussed in chromatography from early on, is illustrated in some detail [2][19]. One can picture the porous medium to be composed of two regions. Namely, the region outside of the particles, where the flow is rapid (mobile region) and the region inside of the porous particles where no flow occurs, but the tracer may enter through diffusion (immobile region). In the simplest version of this two region model one assumes firstorder kinetics to mimic the exchange dynamics between the mobile and immobile region. Denoting the concentration with respect to the pore volume in the mobile and immobile region by $c_{1}(x, t)$ and $c_{2}(x, t)$, respectively, one obtains the following system of equations

$$
\begin{gathered}
\frac{\partial c_{l}}{\partial t}=D \frac{\partial^{2} c_{l}}{\partial x^{2}}-v \frac{\partial c_{l}}{\partial x}-k_{1} c_{l}+k_{2} c_{2} \\
\frac{\partial c_{2}}{\partial t}=k_{l} c_{l}-k_{2} c_{2}
\end{gathered}
$$

where $v=q / \theta_{1}$ and $k_{1}$ and $k_{2}$ are the rate constants for trapping by the immobile region and release into the mobile region, respectively. Several more general formula- 
tions of this model have been discussed recently [21][22]. First, the discussion of the two-region model will be focussed on the limiting cases of slow and rapid exchange. In the case of slow exchange, when the rate of populating the immobile region is very slow $\left(k_{1} \rightarrow 0\right)$, then one simply recovers the convection-dispersion equation discussed earlier and a tracer pulse will move with velocity $v=q / \theta_{1}$, where $\theta_{1}$ is the volume fraction of the mobile region. If, on the other hand, the exchange is very rapid, the concentration in the immobile region is in a steady-state (i.e. $\left.\partial c_{2} / \partial t=0\right)$. One finds that the concentrations in the mobile and immobile phases in the steady-state $c_{1}{ }^{(s)}$ and $c_{2}{ }^{(s)}$ are characterized by an equilibrium constant

$$
\frac{c_{2}^{(s)}}{c_{1}^{(s)}}=\frac{k_{1}}{k_{2}}=K
$$

Inserting this relation into Eqn. 2.10, one again recovers the convection-dispersion equation (Eqn. 2.I), but with a new time variable $t \rightarrow t /(1+K)$. Because of this transformation, the relative spread of the tracer pulse remains unchanged (see Eqn. 2.8 ), but its mean travel time will become longer, namely

$$
<t>=\frac{L}{v}(1+K)
$$

In more physical terms: if the exchange is rapid, there is no difference, whether the tracer moves through the mobile or the immobile region and thus travels with a smaller velocity $v=q / \theta$, where $\theta=\theta_{1}+\theta_{2}$ is the entire volume fraction of the mobile and the immobile region. The equilibrium constant or rather the distribution coefficient between the mobile and the immobile region is then given by $K=\theta_{2} / \theta_{1}$.

Eqns. 2.10 and 2.11 can be solved by Laplace-transform techniques [2][17][19]. Here, the details shall not be discussed, but few important properties of the solution are briefly reviewed. In the intermediate situation, the tracer can either move rapidly in the mobile region but can also become trapped for comparable times in the immobile region. In this situation, one obtains strongly asymmetrical breakthrough curves. One finds that the mean travel time is always given by Eqn. 2.13. The coefficient of variation becomes

$$
\frac{\left\langle\delta t^{2}\right\rangle}{\langle t\rangle^{2}}=\frac{2 D}{v L}+\frac{2 v \tau}{L} \frac{K}{1+K}
$$

where $\tau=\left(k_{1}+k_{2}\right)^{-1}$ is the relaxation time of the exchange between the mobile and immobile region and $D$ is given by Eqn. 2.2 One can see that at low velocities (or rapid kinetics $\tau \rightarrow 0$ ) the tracer has sufficient time to exchange between the mobile and immobile regions. In this case, only the first term of Eqn. 2.14 is important, and the relative width of the tracer pulse decreases with increasing velocity. At higher velocities (or slow kinetics), the exchange process cannot catch up with the flow, and there is a rapidly moving front with a long tail which leads to a increase of the relative width (second term in Eqn. 2.14). Therefore, the relative width of the tracer pulse is minimal at one particular flow velocity. This is of much importance in chromatography, since at this particular flow velocity chromatographic peaks have minimal width which results into an optimal separation performance of the column [20].

Let us now turn to transport of tracers in soils using the concepts from chromatography introduced above. Nevertheless, there are several key differences to be kept in mind. Most importantly, particles of the soil matrix are usually very polydisperse and not homogeneously packed. One has to distinguish transport in groundwater aquifers which are saturated with water and in the unsaturated subsurface zone where pores contain water and air as well. The latter case might lead to very heterogeneous flow structures. Furthermore, cracks and root zones may lead to preferential flow paths resulting in channeling and very rapid flow.

The convection-dispersion equation (Eqn.2.l) may still be applicable under such conditions. As discussed previously, it is essential that the space and time scale considered is much larger than the scale of the underlying random jump process. Unfortunately, the presence of heterogeneities of the soil often requires very large scales for its validity. Often, in a particular experiment one does not really attain the asymptotic region where Eqn. 2.1 is valid but remains in a transient region where time-dependent dispersion coefficient must be considered or the relaxation of other variables included. Often, it is possible to devise simple models such as the two-region model (discussed above) or stochastic stream tube models which may give a much better description on smaller space and shorter time scales. A frequent observation is that with increasing scale of the experiment the observed dispersivity increases [1][23]. It seems that on larger scales one explores heterogeneities which remain hidden on small scales. Such behavior is typical for a situation where the underlying structure of the porous medium never leads to a valid convection-dispersion equation. A well known example of such behavior in physical chemistry is the famous 'ant in the labyrinth' or in more scientific terms, diffusion processes on self-similar media (fractals) [24][25]. It remains to be seen, whether such concepts will become relevant in the discussion of transport processes in natural porous media in the near future.

A few illustrative examples shall be discussed now. We shall not focus on column experiments in the laboratory, since they show dispersivities orders of magnitude smaller than observed in the field. The first example will illustrate the movement of a tracer in groundwater under saturated condi- tions in a sand aquifer. Even though the particles are still fairly monodisperse, they are no longer homogeneously packed as in a chromatography column, but rather their heterogeneous packing density gives rise to correlations on much larger length scales. In a very elaborate and thorough study near a municipal landfill at the Canadian Forces Base, Borden in Canada [10] a pulse of chloride (tracer) was injected into the groundwater aquifer and monitored by several hundred groundwater samplers. The vertically averaged concentrations are shown in Fig. 2. The horizontal projection of the tracer plume ("three-dimensional chromatographic peak') moves with the direction of flow of the groundwater. Using Eyn. 2.4. one can estimate the true velocity of $c a .10$ $\mathrm{cm} /$ day which corresponds quite accurately to the flow velocity of the groundwater. Inserting the characteristic dimensions of the plume of $10-20 \mathrm{~m}$ into Eqns. 2.5 and 2.2 , one finds that the spreading of the plume can be characterized by a dispersivity of the order of several meters. Since sand grains on the site have a diameter below $1 \mathrm{~mm}$, this larger value of the dispersivity must originate from heterogeneous packing of the aquifer. It essential to pay attention to the three-dimensional aspect of the problem. The horizontal layering of the aquifer causes the movement and spreading of the plume to become strongly anisotropic. In fact, such anisotropy effects are also important in a homogeneously packed bed such as a chromatography column. The dispersivity is about one order of magnitude larger in the direction of flow than perpendicular to it. This effect can also be observed in Fig. 2 and results in the elongation of the plume in the direction of its movement. In the vertical direction (not shown in Fig. 2), one observes

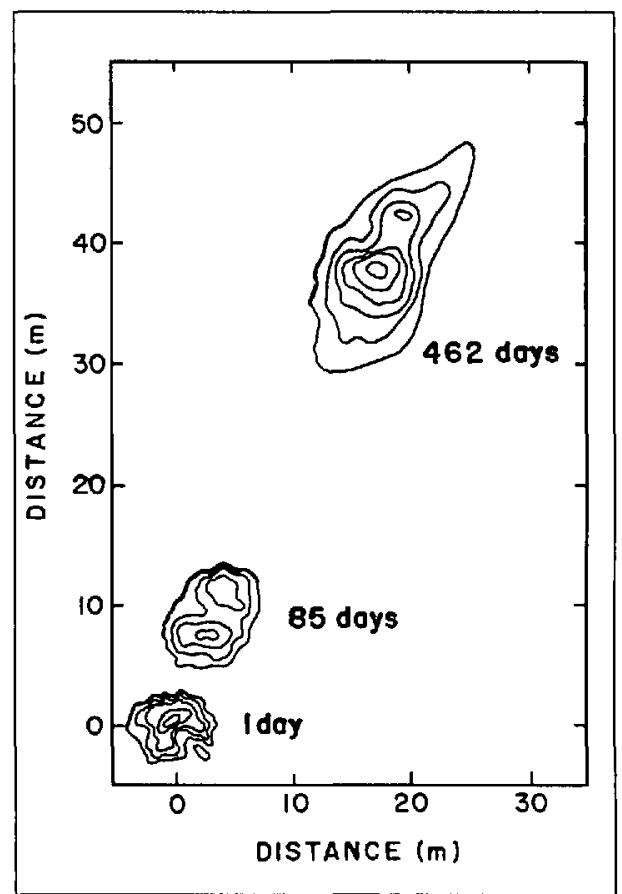

Fig. 2. Horizontally averaged distribution of chloride in the Borden site experiment. The contours depicted start for the $1-\mathrm{d}$ and $85-\mathrm{d}$ plume at $40 \mathrm{mg} / \mathrm{l}$ (interval 40 ) whereas at $10 \mathrm{mg} / \mathrm{l}$ (interval 10) for the $462-\mathrm{d}$ plume. 
a dispersion which is much smaller than in the horizontal direction. E.g., the 462-days tracer plume shown in Fig. 2 has a horizontal extent of tens of meters, but its vertical extent barely exceeds $2 \mathrm{~m}$. The last interesting observation in Fig. 2 is the shape of the plume. In the beginning of the experiment, it appears quite erratic and, at later times, the contours of the plume start to smooth out. Such erratic contours are a strong hint to question the validity of the convection-dispersion equation which will always predicts smooth concentration contours.

In recent literature, there has been much discussion of the quantitative description of the motion of the tracer plume in the Borden site experiment. To account for the strong anisotropy, one has to generalize Eqn.2.1 to three dimensions introducing an anisotropic dispersion tensor. Careful analysis of the data seems to indicate that even after two years, the conditions for the validity of Eqn. 2.1 have not yet been reached. The interested reader may find a useful introduction into this subject in Part 4 of Dagan's book [1].

Consider now the transport of tracers through the subsurface zone. Due to the mostly unsaturated conditions in this zone, which is essentially a three-phase random medium composed of matrix, water and air, dispersion phenomena become even more pronounced. Very similar questions arise in the case of multiphase flow, where oil and water are flowing in a porous medium [7] The detailed description of flow in an unsaturated medium is much more complex. Due to the presence of the void air space, the water channel network will have lot of 'dead end arms' where the tracers will be trapped for a long time. This process may be modeled by the two-region model by adjusting its single exchange time to the average residence time of the tracer. This description will break down for sufficiently low water content. The water-filled pore space may become spatially disconnected (percolation threshold) and has to deal with very broad distributions of residence times [26]. Furthermore, hydrodynamic instabilities may lead to the formation of preferential flow paths ('fingers'). Recently, much research effort is focussed on the transport in such media [25-27].

In spite of all such complications, several field experiments performed in the unsaturated zone indicate that the horizontally averaged concentration profiles show simple structure and may be even described by Eqn. 2.1. Let us now focus on two relevant experiments of this type which investigate transport of tracers in soils.

To illustrate the important aspects, consider a tracer experiment performed in Switzerland [11]. Some results are shown in Fig. 3. A pulse of bromide was applied on the soil surface. Several months later, the soil was dug up along a transect, and the concentration distribution of the tracer was measured. The results in Fig. 3 clearly demonstrate two important aspects. First, due to the spatial heterogeneity of the soil, there is a substantial amount of randomness in the distribution on the tracer in the field. Second, averaging over the different heights (left) one can clearly see a pronounced tracer pulse quite similar to the result of a chromatography experiment. Again, a quick estimate gives the velocity of the pulse of $c a .0 .5 \mathrm{~cm} /$ day and the dispersivity about $2 \mathrm{~cm}$. Because of the nature of this experiment, a 'snapshot' of the distribution is obtained, but one cannot decide, whether the temporal evolution of the peak can be described with Eqn. 2.1. A nice study addressing this point has been performed by Butters et al. [9]. On a field site, which was under intensive irrigation through a sprinkler system, a bromide pulse was applied. The bromide concentration has been periodically recorded in 100 previously installed soil solution samplers. The horizontally averaged concentrations at different depths resembled broad chromatography peaks. The authors have fitted the solution of Eqn. 2.1 to the chromatographic peak at the first depth and used these results to predict the tracer peak deeper in the soil. This study clearly shows that Eqn. 2.I underestimates the spreading of the peak. The results in greater depths could also be fitted using solutions of Eqn. 2.1 but with a dispersivity which increases with increasing depth. This

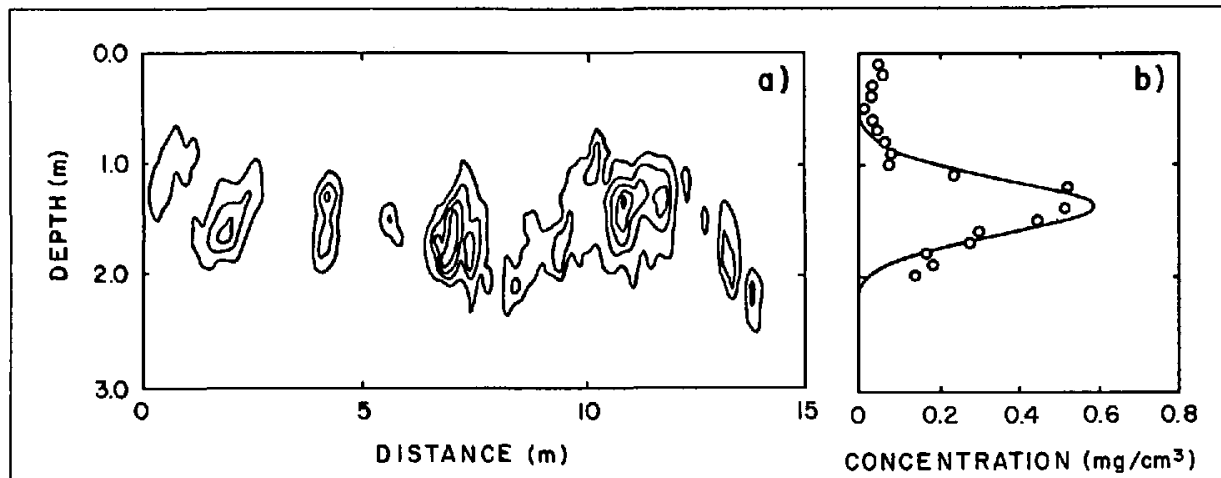

Fig. 3. Infiltration experiment into a unsaturated soil by Schulin et al. [11]. a) Contours of the bromide concentration in the transect. The contours start at $50 \mathrm{mg} / \mathrm{cm}^{3}$ (interval 50). b) Horizontally averaged bromide concentrations (circles). Solid line represents a fit using the convection-dispersion equation. illustrates again the scale dependence of the dispersivity. The authors have devised a different model (stochastic stream tube model) which is able to predict the spread of the peak reasonably well [9].

\section{Transport of Reacting Chemicals}

To address the important features of transport of reactive chemicals, recall the chromatography column and focus on the simplest case of the transport of a single chemical which does neither degrade nor undergo any chemical reactions. The only difference between such a chemical and a conservative tracer is that the chemical may strongly adsorb to the surface of the porous medium. The chemical may be either dissolved and move with the liquid (mobile) or be adsorbed on to the matrix (immobile). The situation is precisely the same as discussed earlier in the context of the two-region model. One just has to re-interpret the meaning of the variables involved. Now, $c_{1}$ is the concentration of the chemical in solution and $c_{2}$ the concentration of the sorbed chemical, both expressed relative to the pore volume. The rate constants $k_{1}$ and $k_{2}$ now correspond to the rate of adsorption and to the rate of desorption, respectively. Of particular interest is the case, when the adsorption equilibrium is reached much faster than the time scale of transport through the chromatography column. In that case, there is equilibrium between the adsorbed species and the relative spread of the peak of the sorbing chemical is the same as for a tracer (see Eqn. 2.14). Its mean travel time is longer, however, and is again given by Eqn. 2.13.

$$
<t>=\frac{L}{v}(1+K)=\frac{L}{v} R
$$

where now the equilibrium constant $K$ (see Eqn. 2.12) corresponds to the distribution coefficient of the adsorption equilibrium. We have also introduced the retardation factor (or retention coefficient) $R=1+K$. Such a sorbing chemical travels slower than a tracer, namely, with a mean velocity $v / R$. The two-region model can be also employed to discuss the effects of slow chemical kinetics. When the adsorption becomes slow, the chemical will move partly like a tracer but show a long time tail due to the adsorption process which leads to strongly asymmetric breakthrough curves [28][29]

The analogy to the two-region model strictly holds in the case of a linear adsorption isotherm (or constant distribution coefficient) only. Experimentally one often observes nonlinear adsorption isotherms which are usually fitted by Langmuir or Freundlich expressions [30]. The nonlinear nature of the adsorption equilibrium has profound and interesting consequences for transport [12][31]. Consider the Langmuir adsorption isotherm as an example. Picture the surface composed of identical surface sites $S$ where e.g. a dissolved metal ions $M$ adsorb and 
form a metal-surface complexes SM. This reaction can be described by a simple chemical equilibrium

$$
\mathrm{M}+\mathrm{S} \rightleftharpoons \mathrm{SM}
$$

with an equilibrium constant

$$
K_{L}=\frac{c_{S M}}{c_{S} c_{M}}
$$

Observing that the total concentration of sites is constant, namely $c_{0}=c_{S M}+c_{S}$, using Eqn.3.3 one obtains the Langmuir adsorption isotherm

$$
c_{S M}=c_{0} \frac{K_{L} c_{M}}{1+K_{L} c_{M}}
$$

This isotherm is plotted in Fig. 4. At low concentrations, it behaves linearly while it saturates at high concentrations.

How does the breakthrough of a solute which follows the Langmuir (or a similar) isotherm look like? First of all, the response of the column is no longer linear and will depend on the concentration of the solute. Since the Langmuir isotherm shows saturation, the partition coefficient decreases with increasing concentration and thus leads to a decreasing retardation factor. Thus, one expects that the higher the concentration the earlier the breakthrough. A second, quite an intriguing aspect of the nonlinearity of the system manifests itself that the shape of the breakthrough curve can be deduced from a simple rule by $F . M$. Golden which is known in the French-speaking community as "la règle de la ficelle' [31]. This rule is illustrated in Fig. 4. Imagine a string attached to the starting point on the isotherm (origin $\mathrm{O}$ for the adsorption step and the marked point A for the desorption step). Holding the string, one approaches the end point of the process on the isotherm ( $A$ for adsorption, $O$ for desorption), in counter-clockwise direction. If now the string lies flat on the isotherm, a broadening front is observed (desorption), while when the string is freely stretched between the starting and end point a sharp front is observed (adsorption). This rule can

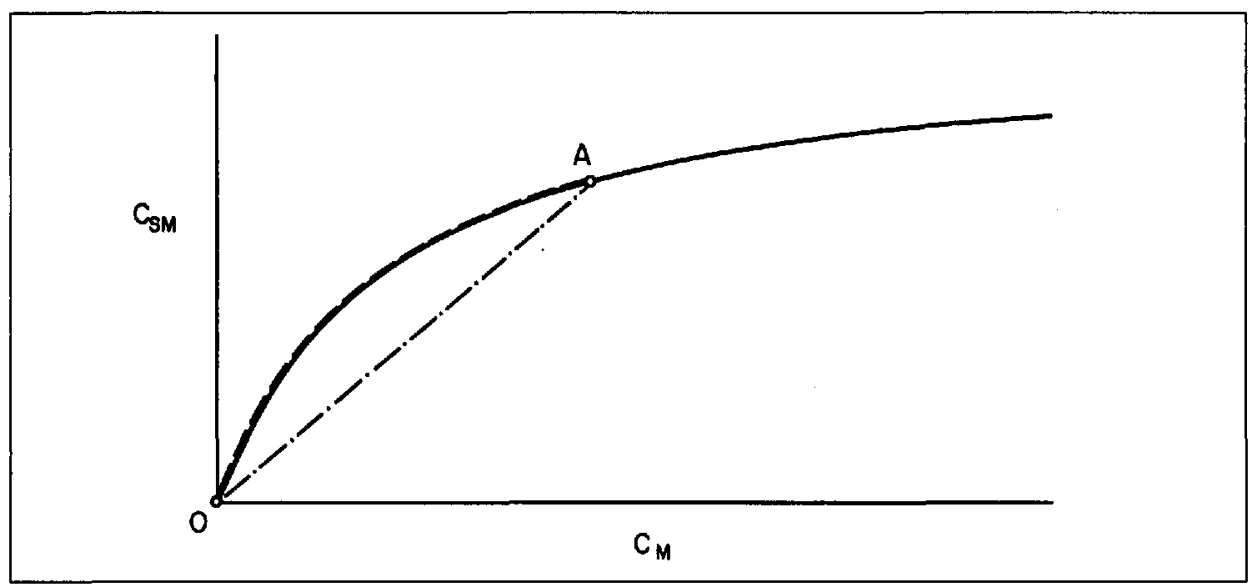

Fig. 4. Plot of nonlinear adsorption Langmuir isotherm (Eqn.3.4 solid line) and illustration of the Golden rule (see text for details). The string connects the points $\mathrm{O}$ and $\mathrm{A}$ during adsorption (dash dotted) and desorption (dashed).

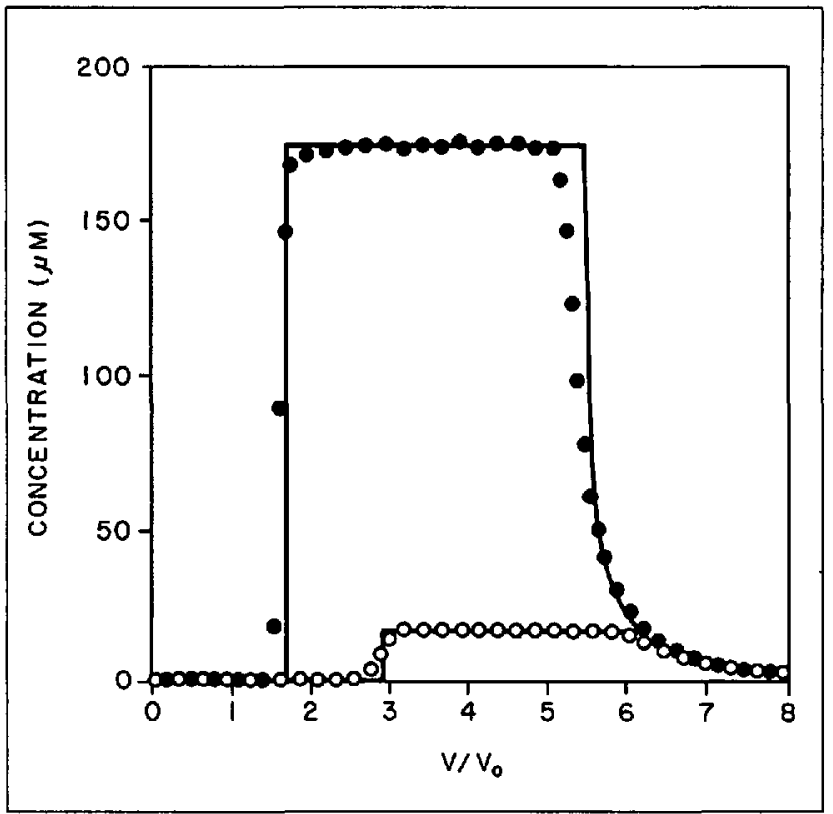

Fig. 5. Breakthrough curves of cadmium at pH 8 through a sand $\mathrm{col}$ umn with desorption with pure buffer solution (after $V / V_{0}=4$ ). One can observe several features of breakthrough curves typical for nonlinear sorption: concentration dependence of the breakthrough, formation of a self-sharpening front during adsorption and of a concentration-independent diffuse front during desorption [32].

be understood in more physical terms as follows. In the case of the desorption process, one starts at high concentrations (retardation factor being small) and proceeds to low concentrations (retardation factor being large). This increase of the retardation will cause a substantial broadening of the front. In the adsorption process, on the other hand, one starts with a large retardation ending with a small retardation. This leads to an instability and to the formation of a selfsharpening front which is a traveling nonlinear wave (soliton). These features are illustrated in Fig. 5 where experimental data of the breakthrough of $\mathrm{Cd}$ in a buffer solution of $\mathrm{pH} 8$ through a sand column are shown [32]. The adsorption isotherm is similar to Fig. 4 and highly nonlinear which results to the strong concentration dependence of the breakthrough shown in Fig. 5. During the adsorption process, the front is narrow (selfsharpening), whereas during the desorption one observes a broad (diffuse) front with concentration independent tailing. To stress this 'chemical dispersion', the solid lines shown in Fig. 5 correspond to a calculation using the experimental adsorption isotherm but neglegting the dispersion of the column.

Until now, it has been focussed on the

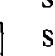
complexation in the aqueous phase, adsorption-desorption processes, ion-exchange, precipitation reactions, gas solubility, and redox equilibria. We refer to recent reviews for the details of the procedures and the nowadays available computer codes for the solution of such problems [33-35]. The treatment of effects of slow chemical kinetics in such a general framework is not yet well developed.

In the general case, several fronts which may be either self-sharperning or diffuse will be observed [12]. Usually, one has to expect a front traveling like a tracer, the socalled normality wave. To illustrate these concepts, consider a few examples. First, a 
simple exchange between monovalent and divalent cations such as $\mathrm{Na}^{+}$and $\mathrm{Ca}^{2+}$ on clay minerals described by a chemical equilibrium

$$
\mathrm{Ca}^{2+}+2 \mathrm{Na}_{\mathrm{S}}^{+} \rightleftharpoons \mathrm{Ca}_{\mathrm{S}}^{2+}+2 \mathrm{Na}^{+}
$$

where the subscript $S$ denotes the sorbed species. An illustrative column experiment has been performed by Jauzein et al. [35][36]. The concentrations of the cations at the outlet are shown Fig. 6. The soil column, initially saturated with $\mathrm{Ca}^{2+}$ and washed with $\mathrm{H}_{2} \mathrm{O}$ was subjected to a step input of $20 \mathrm{~mm}$ $\mathrm{NaCl}$ solution and after 4 pore volumes (arrow in Fig. 6) the feeding solution changed to $5 \mathrm{~mm} \mathrm{CaCl}_{2}$. After one-pore volume, calcium exits the column which corresponds to a normality wave. This wave is immediately followed by a diffuse front of the exchange of calcium by sodium. After 5 pore volumes, one again observes a normality wave which is now followed by a self-sharpening front (after 6.5 pore volumes) of the exchange of sodium by calcium. The results of a numerical simulation are also shown in Fig. 6. All chemical equilibrium constants involved have been determined in different batch experiments and, therefore, there are no adjustable parameters in the calculation. Fig. 6 illustrates that one is able to predict quantitatively all patterns of this column experiment.

It is possible to use the same type of approach to predict quite complex features of a multi-component reactive transport involving many reacting components. The result of such a simulation is confronted with experiments data and is shown in Fig. 7. One should note that again only one parameter (the dispersivity) is fitted. All others, such as the chemical equilibrium constants, have been obtained from independent batch experiments.

Finally, consider field studies of reactive solute transport. Unfortunately, there are very few experimental results available. As the first example will serve again the Borden site experiment [37]. During the application of the tracer pulse, several non-conservative solutes (chlorinated hydrocarbons) were also injected. In Fig. 8, we present the time evolution of the concentrations of chloride, $\mathrm{CCl}_{4}$ and tetrachloroethylene in a bore hole $5 \mathrm{~m}$ downstream. This figure illustrates beautifully that the groundwater aquifer acts as a giant (though somewhat impractical) chromatography column which properly separates the three components injected. From the position of the peaks, one may estimate

Fig. 6. Experimental results and simulation of a column experiment with calcium-sodium exchange [351[36] Sodium (full circles) calcium (open circles). See text for details.

Fig. 7. Experimental results (dots) and simulation (full lines) of a column experiment with ion-exchange and precipitation reactions. Sodium ions (open circles), calcium ions (full circles) and an anionic surfactant (triangles) [36]
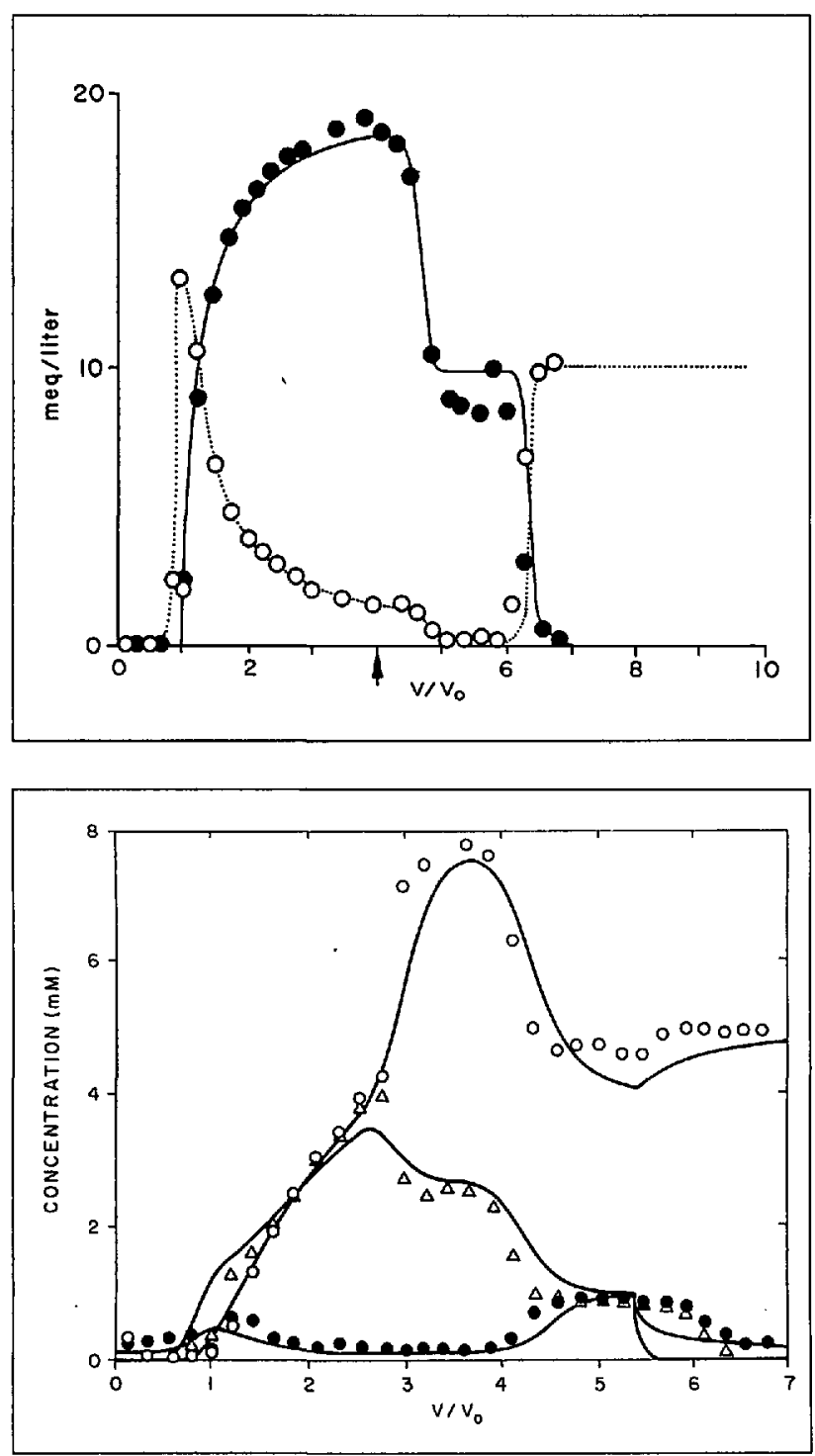

the retardation factors of 1.8 for $\mathrm{CCl}_{4}$ and 3.3 for tetrachloroethylene. These values are in reasonable agreement with estimates based on measurements of partition coefficients obtained from laboratory-batch experiments [37]. Yet one finds, that with increasing traveled distance the retardation factors increase. This behavior has been also observed on different sites and might be connected with the spatial heterogeneity of the adsorption parameters in the field [38]. Presently, there is much activity investigating the connection between spatial heterogeneity of soils and aquifers, the corresponding adsorption properties and their effects on the movement of non-conservative tracers [39].

As a final example of multi-component transport in the field, the results of Valocchi et al. [14] are discussed where multi-component ion-exchange phenomena could be observed in the field. Into a groundwater aquifer, water of lower ionic strength has been injected, and concentrations of several ions have been monitored at a pumping well several meters away. The average velocity of the groundwater is $1 \mathrm{~m} / \mathrm{h}$ and the dispersivity of the aquifer $3 \mathrm{~m}$. The simulated time development of the cation concentrations $\left(\mathrm{Na}^{+}, \mathrm{Ca}^{2+}\right.$, and $\left.\mathrm{Mg}^{2+}\right)$ and a tracer (hypothetical monovalent counter-ion $\mathrm{X}^{-}$) are compared with the measured field data in Fig. 9. The numerical results are in good agreement with the experimental data [14][33]. The results of this experiment also allow simple interpretation in terms of chromatographic waves. The first decrease after roughly $10 \mathrm{~h}$ corresponds to the normality wave (tracer). The second and third wave (near 500 and $2000 \mathrm{~h}$ ) correspond to the two following ion exchange reactions

$$
\begin{aligned}
& \mathrm{Ca}^{2+}+2 \mathrm{Na}_{\mathrm{S}}^{+} \rightleftharpoons \mathrm{Ca}_{\mathrm{S}}^{2+}+2 \mathrm{Na}^{+} \\
& \mathrm{Ca}^{2+}+\mathrm{Mg}_{\mathrm{S}}^{2+} \rightleftharpoons \mathrm{Ca}_{\mathrm{S}}^{2+}+\mathrm{Mg}^{2+}
\end{aligned}
$$

First, the exchange sites have adsorbed excess sodium, which comes to the equilibrium value after the second wave. The third wave corresponds to the establishment of the equilibrium between calcium and magnesium.

\section{Conclusion}

This review has emphasized the impact of theories of chromatography upon the understanding of transport of chemicals in natural porous media such as soils and groundwater aquifers. We have discussed the transport of tracers and realized that the dispersion process in a chromatography column is much less dispersive than in a heterogeneous soil. The large spreading of tracers originates from the long correlation lengths in the soil structure. In analogy to chromatography, where adsorption of chemicals leads to different travel velocities in the column, the same process causes retardation of sorbing chemicals in soils. The 


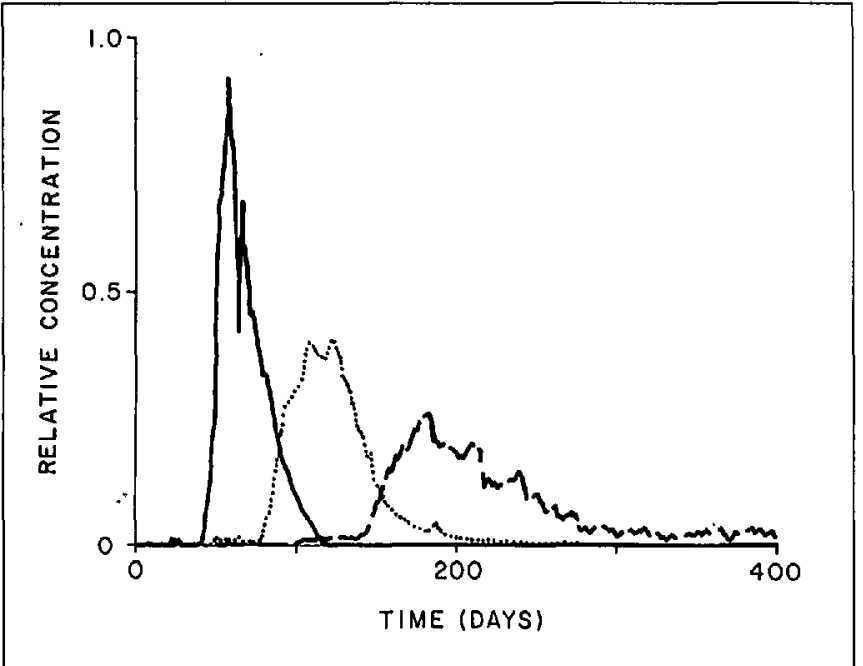

Fig. 8. Time record of concentrations of chloride (full line), $\mathrm{CCl}_{4}$ (dotted line), and tetrachloroethylene (dashed line) in a sampling well of the Borden site experiment [37]

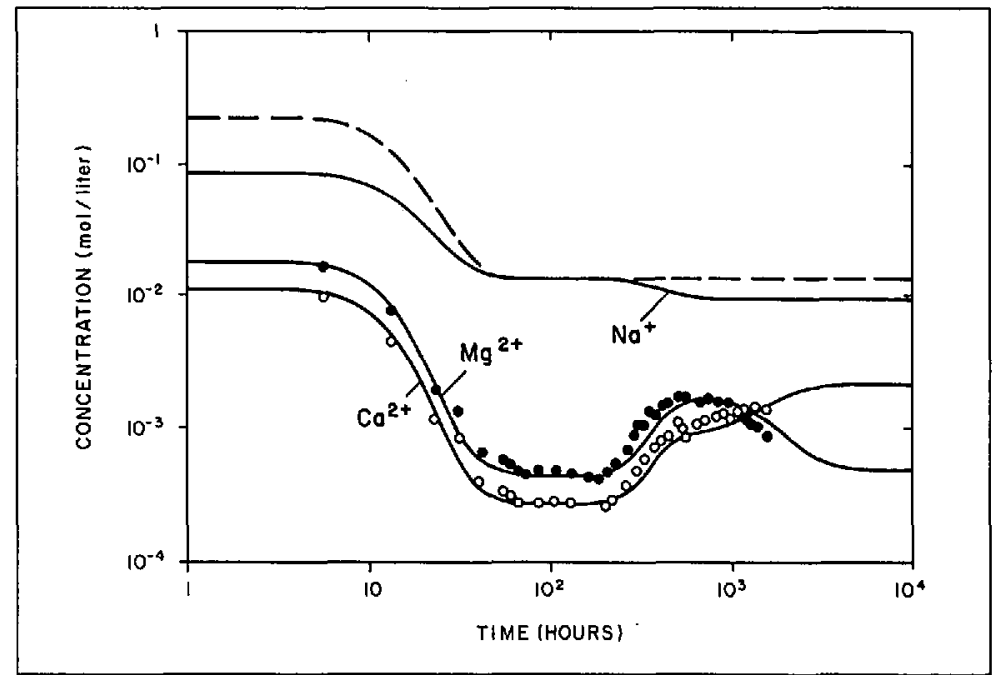

Fig. 9. Concentration dependence of cation concentrations (dots) in a ficld cxperime'nt of Valocchi et al. [14]. Lines are results of numerical simulations. Solid lines represent exchangeable cations, and dashed line corresponds to the jonic strength (sum of anions. tracer). interaction of several components may lead to quite complicated sequences of solute fronts (multi-component transport).

Consider briefly some novel aspects in this field. These specialized topics will not be discussed in detail, but let us rather draw attention upon some interesting ideas. First appealing field is the transport of organic chemicals such as hydrocarbons or chlorinated hydrocarbons which are sparingly soluble in water. As long as they are volatile, the air space in the unsaturated zone represents an important transport pathway [6]. Large amounts of such organic compounds will accumulate in the soil matrix as a separate phase, such that one is confronted with a medium composed of four different regions, namely solid matrix, air, water, and oil. Depending on the density, the organic phase may float on the groundwater table or sink below the groundwater to the bedrock. For particular viscosity ratios, the infiltration may proceed in a very irregular way (viscous fingering). Very similar processes are of much interest in tertiary oil recovery [7]. A second interesting topic is the scavenging of pollutants by small colloidal particles [8]. For highly sorbing pollutants such as heavy metals or radionuclides, one often observes a rapid movement of a very small fraction of the chemical [28]. Such movement is explained by the presence of small and mobile particles (organic or inorganic) in the groundwater. The pollutant usually strongly adsorbs onto the particles and the particle, thus, may act as a carrier for the pollutant. There is still not much known about the importance of this process in realistic situations.

In summary, application of concepts from chromatography to soil-solute transporthave been reviewed. The aim was to reveal the fascination of the interplay between physics and chemistry together with the applied sciences such as aquatic chemistry, chemical engineering, hydrology, and soil sciences in this field. In our view, much better under- standing and progress could be made, when more active communication between researchers in these disciplines would take place.

We thank H.Flïhler. S. Koch, A. Scheidegger, and R. Schulin for enjoyable discussions. Particular thanks go to $A$. Zysset and $F$. Stauffer for allowing us to use their transport code and to $C$. Bürgisser for making her results available prior to publication.

Received: June 14, 1991

[1] G. Dagan, 'Flow and Transport in Porous Formations', Springer, New York, 1989.

[2] W. A. Jury, K. Roth, 'Transfer Functions and Solute Movement through Soil', Birkhäuser, Basel, 1990.

[3] S. Lesage, R. E. Jackson, M. W. Priddle, P. G Riemann, Environ. Sci. Technol. 1990, 24, 559; D. S. MacFarlane, J. A. Cherry, R. W. Gilham, E A. Sudicky, J. Hydrol. 1983, 63,1.

[4] G. Sposito, A. L. Page, in 'Metal ions in biological systems', Ed. H. Siegel, M. Dekker, New York, 1984.

[5] R. V. Nicolson, J. A. Cherry, E. J. Reardon, J. Hydrol. 1983, 63, 131 .

[6] W. E. Spencer, M. M. Cliath, W. A. Jury, L. Z Zhang, J. Environ. Qual 1988, 17, 504.

17] F. Schwille, in 'Pollutants in Porous Media', Ed. B. Yaron et al., Springer, New York, 1984.

[8] D. A. Backhus, P. M. Gschwend, Environ. $S c i$ Technol. 1990, 24, 1214; R. W. Harvey, S. P. Garabedian, ibid. 1991, 25, 178

[9] G. Butters, W. A. Jury, F. F. Ernst, Water Resour Res. 1989, 25, 1575, 1583

[10] D. M. Mackay, D. L. Freyberg, P. V. Roberts, J. A. Cherry, Water Resour. Res. 1986, 22, 2017.

[11] R. Schulin, M. T. van Genuchten, H. Flühler, P Ferlin, Water Resour. Res. 1987, 23, 1785.

[12] F. Helfferich, G. Klein, 'Multicomponent Chromatography', Dekker, New York, 1970.

[13] D. R. Nielsen, J. W. Biggar, Soil Sci. Soc. Am. Proc. 1961, 25, 1

[14] A. Valocchi, R. L. Street, P. V. Roberts, Water. Resour. Res. 1981, 17, 1517 .

[15] S. Berry, S. Rice, J. Ross, 'Physical Chemistry' John Wiley, New York, 1985.

[16] J. Bear, 'Dynamics of Fluids in Porous Media', Elsevier, New York, 1972

[17] J. Villermaux, in 'Percolation Processes', Eds. A. E. Rodriques and D. Tondeur, Sijthoff, Amsterdam, 1981.
[18] R. Aris, "The mathematical theory of diffusion and reaction in permeable catalysts', Clarendon Press, Oxford, 1975; M. T. van Genuchten, P. J. Wierenga, Soil Sci. Soc. Am. J. 1976, 40, 473.

[19] L. Lapidus, N. Amundson, J. Phys. Chem. 1956. $56,984$.

[20] D. A. Skog, D. M. West, 'Principles of Instrumental Analysis', Sanders, Holt, 1980.

[21] M. Sardin, D. Schweich, F. J. Leij, M. T. van Genuchten, Water. Resour. Res. 1991, in press.

[22] R. M. Nicoud, D. Schweich, Water Re'sontr. Res. 1989, 25, 1071.

[23] A. Lallemand-Barres, P. Peaudecerf, Bull. $B R C M$ Sect. III 1978, 4, 277; L. W. Gelhar, Water. Resour. Res. 1986, 22, $135 S$.

[24] P. G. de Gennes, J. Phys.(Paris) Letr. 1976, 37. L1.

[25] N. Boccara, M. Daoud, 'Physics of Finely Divided Matter', Springer, New York, 1985.

[26] P. G. de Gennes, J. Fluid. Mech. 1983, 136, 189.

[27] M. Buès, M. Aachib, Exp. Fluids 1991, $11,25$.

[28] P. Behra, Geoderma 1986, 38, 209.

[29] H. M. Selim, R. Schulin, H. Flühler, Soil Sci.Soc. Am. I. 1987, 5/,876.

[30] R. A. Freeze, J. A. Cherry, 'Groundwater', Prentice-Hall, Englewood Cliffs, 1979); D. G. Kinniburgh, Environ.Sci. Technol. 1986, 20,895; C. C. Travis, E. L. Etnier, I. Environ. Qual. 1981. 10,8 .

[31] D. Schweich, M. Sardin, J. Hydrol. 1981, 50, I.

[32] C. Bürgisser, M. Borkovec, H. Sticher, to be published.

[33] A. Zysset, P. Behra, F. Stauffer T. Dracos, ERCOFTAC Workshop on 'Chemical Reactions in Turbulent Fluids', Lausanne, 1991; P. Behra, A. Zysset, L. Sigg, F. Stauffer, EAWAG-New's 1990, $28 / 29,6$.

[34] D.C. Mangold, C. F. Tsang, Rev. Geophys. 1991 , 29, 51; G. T. Yeh, V. S. Tripathi, Water, Reserer. Res. 1989, 25, 93; P. C. Lichtner, Gerolhim. Cosmochim. Acta 1985, 49, 779 .

[35] M. Jauzein, C. Andr'e, R. Margrita, M. Sardin, D. Schweich, Geoderma 1989, 44, 95

[36] R. Krebs, M. Sardin, D. Schweich, AIChE I. 1987, 33. 1371; M. Sardin, R. Krebs, D. Schweich, Geoderma 1986, 38, 115.

[37] D. M. Mackay, D. L. Freyberg, P. L. McCarty, P. V. Roberts, Water Resour. Res. 1986, 22, 2047; G. P. Curtis, P. V. Roberts, M. Reinhard, ibid. 1986, 22, 2059.

[38] P. Ackerer, P. Behra, in Proc. Int. Conf. 'Impact of physico-chemistry on the study, design and optimisation of processes in natural porous media', Nancy, 1987.

[39] Z.J.Kabala, G.Sposito, WaterResour.Res. 1991 27,341 . 